\title{
Utility of Whole Blood Thiamine Pyrophosphate Evaluation in TPK1-Related Diseases
}

\author{
Enrico Bugiardini ${ }^{1,2}{ }^{-}$, Simon Pope ${ }^{3}$, René G. Feichtinger ${ }^{4}\left(\mathbb{0}\right.$, Olivia V. Poole ${ }^{1,2}$, \\ Alan M. Pittman ${ }^{2}$, Cathy E. Woodward ${ }^{5}$, Simon Heales ${ }^{3}$, Rosaline Quinlivan ${ }^{1,2,6}$, \\ Henry Houlden ${ }^{1,2}$, Johannes A. Mayr ${ }^{4}$, Michael G. Hanna ${ }^{1,2}$ and Robert D.S. Pitceathly ${ }^{1,2, * \mathbb{C}}$ \\ 1 MRC Centre for Neuromuscular Diseases, UCL Queen Square Institute of Neurology and National Hospital \\ for Neurology and Neurosurgery, London WC1N 3BG, UK \\ 2 Department of Neuromuscular Diseases, UCL Queen Square Institute of Neurology, London WC1N 3BG, UK \\ 3 Neurometabolic Unit, National Hospital for Neurology and Neurosurgery, London WC1N 3BG, UK \\ 4 Department of Pediatrics, University Hospital Salzburg, Paracelsus Medical University, 5020 Salzburg, Austria \\ 5 Neurogenetics Unit, National Hospital for Neurology and Neurosurgery, London WC1N 3BG, UK \\ 6 Dubowitz Neuromuscular Centre, Great Ormond Street Hospital, London WC1N 3JH, UK \\ * Correspondence: r.pitceathly@ucl.ac.uk; Tel.: +44-(0)2031087527
}

Received: 30 May 2019; Accepted: 3 July 2019; Published: 8 July 2019

\begin{abstract}
TPK1 mutations are a rare, but potentially treatable, cause of thiamine deficiency. Diagnosis is challenging given the phenotypic overlap that exists with other metabolic and neurological disorders. We report a case of TPK1-related disease presenting with Leigh-like syndrome and review the diagnostic utility of thiamine pyrophosphate (TPP) blood measurement. The proband, a 35-year-old male, presented at four months of age with recurrent episodes of post-infectious encephalopathy. He subsequently developed epilepsy, learning difficulties, sensorineural hearing loss, spasticity, and dysphagia. There was a positive family history for Leigh syndrome in an older brother. Plasma lactate was elevated $(3.51 \mathrm{mmol} / \mathrm{L})$ and brain MRI showed bilateral basal ganglia hyperintensities, indicative of Leigh syndrome. Histochemical and spectrophotometric analysis of mitochondrial respiratory chain complexes I, II+III, and IV was normal. Genetic analysis of muscle mitochondrial DNA was negative. Whole exome sequencing of the proband confirmed compound heterozygous variants in TPK1: c. 426G>C (p. Leu142Phe) and c. 258+1G>A (p.?). Blood TPP levels were reduced, providing functional evidence for the deleterious effects of the variants. We highlight the clinical and bioinformatics challenges to diagnosing rare genetic disorders and the continued utility of biochemical analyses, despite major advances in DNA sequencing technology, when investigating novel, potentially disease-causing, genetic variants. Blood TPP measurement represents a fast and cost-effective diagnostic tool in TPK1-related diseases.
\end{abstract}

Keywords: mitochondrial diseases; TPK1; thiamine pyrophosphate; Leigh syndrome; thiamine deficiency

\section{Introduction}

Genetic disorders of thiamine transport and metabolism are a rare but treatable cause of thiamine deficiency that usually present during childhood [1]. Four genetic defects are reported; three present with a predominantly neurological phenotype (SLC19A3, SLC25A19 and TPK1) and one with multisystem disease (SLC19A2), including megaloblastic anaemia, thrombocytopenia, diabetes, and hearing loss. Thiamine pyrophosphokinase 1 (hTPK1, EC2.7.6.2), encoded by TPK1, converts free thiamine to active thiamine pyrophosphate (TPP). Early recognition is therefore crucial given the potential benefits of thiamine supplementation. However, diagnosis of TPK1-related diseases is often 
delayed because of the clinical overlap with other metabolic diseases, including Leigh syndrome. Consequently, patients often undergo numerous investigations, including muscle and skin biopsies, prior to receiving a molecular diagnosis. Here, we describe a case of TPK1-related disease that highlights the potential applications of whole blood TPP measurement for diagnosis and subsequent biochemical monitoring following thiamine supplementation.

\section{Case Presentation}

The proband, the fourth child of healthy unrelated parents, was an uncomplicated pregnancy and delivery and reached all early motor milestones without delays. An older brother had been diagnosed with Leigh syndrome in the early childhood, while two older siblings were healthy. He presented with a post-viral encephalopathy aged four months following varicella-zoster infection and at nine months sensorineural hearing loss was detected. He subsequently experienced recurrent episodes of encephalopathy associated with intercurrent infection and was diagnosed with learning difficulties and seizures. At 22 years he was evaluated in a specialist mitochondrial clinic. At this time, he required fulltime use of a wheelchair and, despite improved seizure control with dual anticonvulsant therapy, continued to experience daily episodes of focal impaired awareness. Neurological examination revealed optic disc atrophy and sensorineural hearing loss. In limbs, there was a spastic increase in tone, hyper-reflexia, and bilateral extensor plantar responses. Laboratory tests, including CPK, fasting acylcarnitine profile, and very long chain fatty acids, were normal. Plasma lactate was elevated ( $3.51 \mathrm{mmol} / \mathrm{L}$, reference range $0.5-2.22 \mathrm{mmol} / \mathrm{L}$ ). Brain MRI revealed bilateral, symmetrical T2 hyperintensities in the corpus striatum and severe cerebellar and moderate brainstem volume loss, without signal abnormalities (Figure 1). The cervical spinal cord displayed normal imaging appearances.
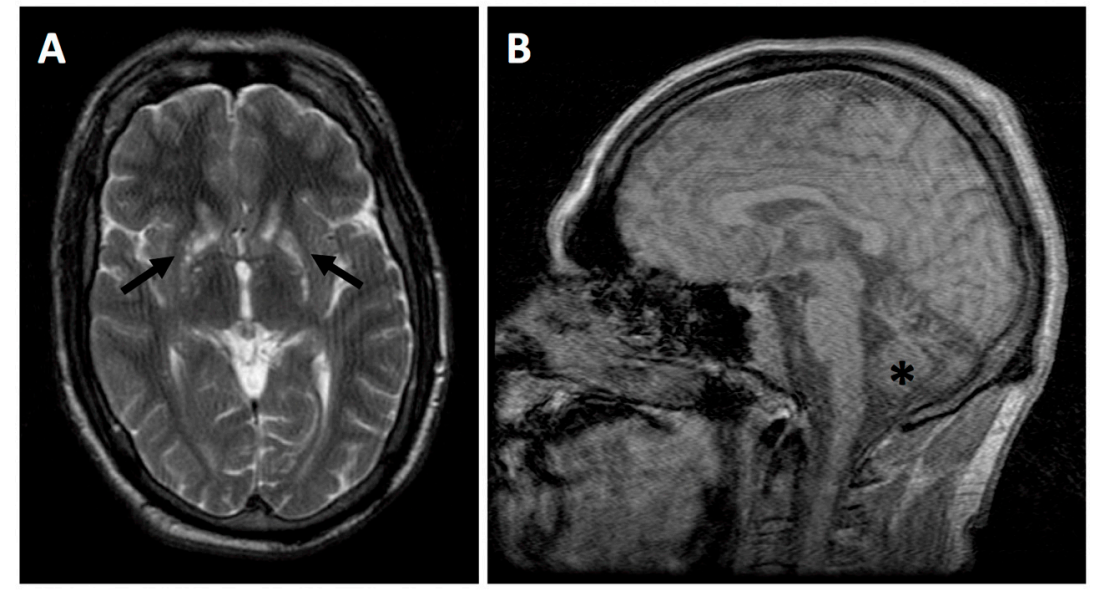

Figure 1. Brain MRI. (A) Axial image demonstrates bilateral, symmetrical T2-weighted high signal intensities within the corpus striatum (arrows). (B) Sagittal image shows cerebellar atrophy (asterisk).

Pyruvate dehydrogenase complex (PDC) activity in cultured fibroblasts, and histological, histochemical, and mitochondrial respiratory chain enzyme analyses of skeletal muscle tissue, was normal. Sanger sequencing of the entire mitochondrial genome and molecular analysis for large scale mitochondrial DNA rearrangements using long range PCR were negative. Whole exome sequencing was performed using the proband's genomic DNA extracted from peripheral leukocytes (Appendix A). Variants with a minor allele frequency (MAF) of $\geq 0.01$ reported in gnomAD [2] and 1000 Genomes databases, and synonymous and deep intronic variants, were excluded. Recessive and X-linked inheritance patterns were prioritised (Supplementary Figure S1). First, a list of genes encoding mitochondrial-localized proteins ( $n=1158$, Mitocarta) [3] was analysed. A variant in NDUFA1 (c.94G>C; p.Gly32Arg), previously reported in two cousins with a progressive neurodegenerative disorder associated with complex I deficiency [4], was identified. However, given the relatively high MAF reported in gnomAD (1189/205094, 408 hemizygotes, MAF 0.005797), and the normality of mitochondrial 
respiratory chain complex I enzyme activity in the proband's muscle tissue, additional screening of variants detected in all remaining coding genes was undertaken. This revealed two missense mutations in TPK1: c.426G>C; p.Leu142Phe (MAF 0.000028 in gnomAD), which exists within a highly conserved region within the catalytic domain of hTPK1 (Figure 2A); and c.258+1G $>$ A, residing in the donor splice site of intron 5 (Supplementary Figure S2). Segregation studies in affected and unaffected family members confirmed that both variants were in trans in the proband and his affected brother. Importantly, the NDUFA1 variant failed to segregate with the disease (Figure 2B). TPP levels in the proband were low ( $35 \mathrm{nmol} / \mathrm{L}$, reference range 67-265), providing biochemical support for the pathogenic effects of the variants, while hTPK1 steady state protein levels were reduced (Figure 2C), consistent with previous reports of TPK1 mutations (see Appendix A for detailed methods) [5]. No additional bands were detected during the western blot analysis, indicating that the splicing variant most likely causes an unstable mRNA molecule. Oral thiamine, $100 \mathrm{mg}$ twice a day, was commenced. Following the most recent assessment, TPP levels had increased to $59 \mathrm{nmol} / \mathrm{L}$ and lactate had normalized $(1.74 \mathrm{mmol} / \mathrm{L})$. However, no meaningful clinical improvement was observed.

A

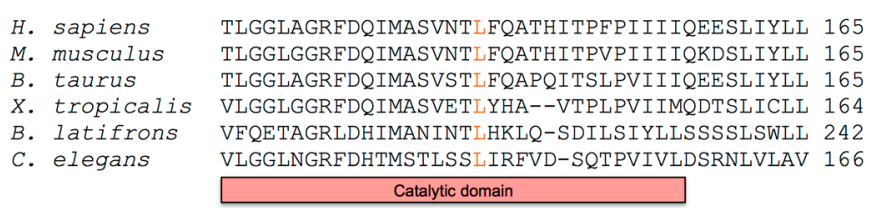

B

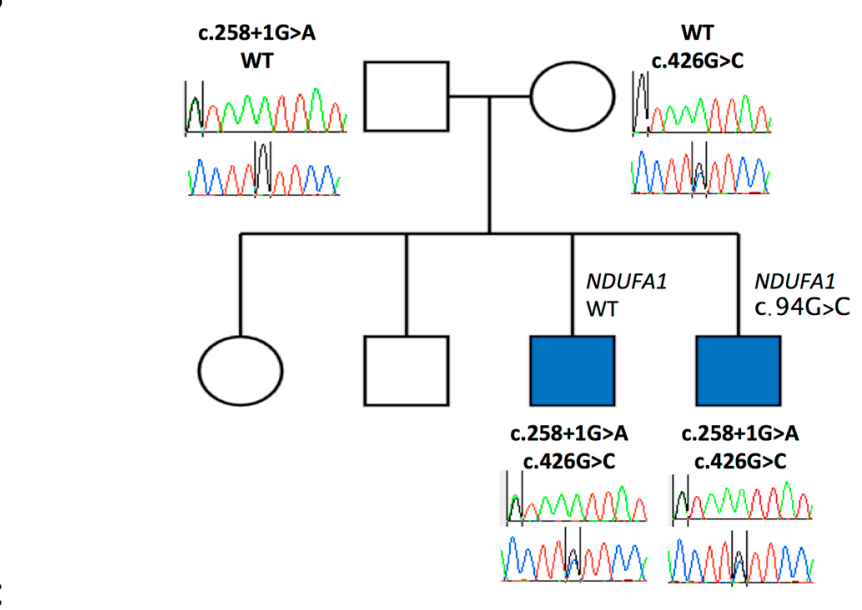

C

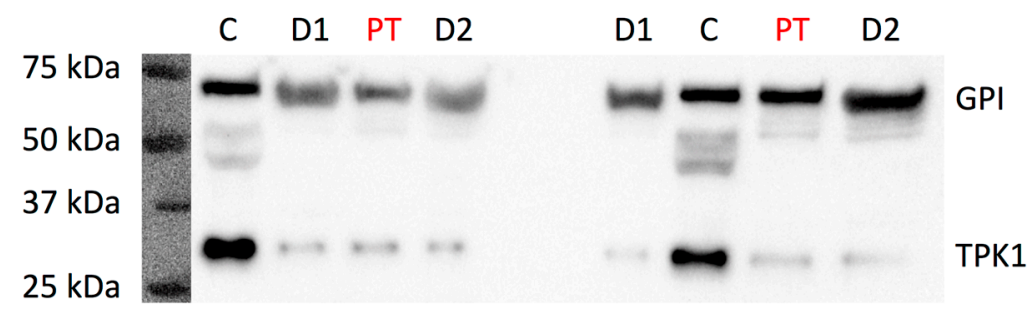

Figure 2. Domain and amino acid conservation for the c.426G >C; p.Leu142Phe variant, and segregation studies and impact on thiamine pyrophosphokinase 1 (TPK1) protein steady state levels of both TPK1 variants. (A) Conservation of hTPK1 amino acid sequence between species. The L142 residue is highly conserved and resides within the catalytic domain of the protein. (B) Family pedigree and segregation analysis. NDUFA1 variant: c.94G>C. TPK1 variants (bold): c.426G >C and c.258+1G>A. (C) Steady state hTPK1 protein levels in patient fibroblasts. Abbreviations: GPI, glucose-6-phosphate isomerase (loading control); C, healthy control; D1 and D2, positive controls-previously reported as P2 and P5, respectively (Mayr et al., 2011) [6]; PT, proband in present study. 


\section{Discussion}

We report a new case of TPK1-related disease and highlight the clinical and diagnostic challenges of these potentially treatable disorders.

Inherited diseases of thiamine metabolism impair the transport and activation of thiamine. Thiamine exists in various phosphorylated states: unphosphorylated thiamine; thiamine monophosphate (TMP); thiamine diphosphate/pyrophosphate (TPP); and thiamine triphosphate (TTP) (Figure 3A). TPP is the active form of thiamine and acts as an important co-factor for several enzymes that play a major role in energy metabolism, including pyruvate dehydrogenase complex. Four primary genetic defects of thiamine metabolism are reported which impair different stages of thiamine transport or activation (Figure 3B). These include: (1) thiamine responsive megaloblastic anaemia (SLC19A2); (2) thiamine metabolism dysfunction syndrome 2 (biotin- or thiamine-responsive encephalopathy type 2, SLC19A3); (3) thiamine metabolism dysfunction syndrome 5 (episodic encephalopathy type, TPK1); and (4) thiamine metabolism dysfunction syndrome 4 (progressive polyneuropathy type, SLC25A19).

SLC19A3-, TPK1-, and SLC25A19-related disorders present primarily with nervous system phenotypes that are difficult to distinguish from isolated mitochondrial encephalopathy. However, early recognition is important so that treatment with thiamine can be initiated. To facilitate this process, diagnostic criteria have recently been established [5]. Unfortunately, given their rarity, only limited data concerning TPK1-related diseases exists; only 13 patients are reported [5-10]. The majority of cases exhibited early onset, recurrent encephalopathic episodes, frequently triggered by infection, followed by ataxia, epilepsy, pyramidal signs, and dystonia. One family presented with progressive dystonia without recurrent encephalopathic episodes [10]. The clinical course of the disease tends to be severe, with high levels of physical disability and reduced lifespan; of the 13 patients, three died at 29 months, three, and eight years. There is no clear genotype-phenotype correlation and the response to thiamine supplementation is variable [5].

Our case highlights the diagnostic challenges in TPK1-related diseases. The clinical phenotype was compatible with Leigh syndrome, prompting investigations to exclude a mitochondrial disorder. However, muscle histochemistry and respiratory chain enzyme activity were normal and consistent with previous reports of TPK1-related disease. An unbiased next generation sequencing (NGS) approach helped identify two missense TPK1 variants: c.426G $>C$ (p.Leu142Phe) and c.258+1G $>$ A (p.?). The c.426G $>C$ variant has been reported once in ClinVar and considered as a likely pathogenic (RCV000255057). Interestingly, the c.425T >C variant affecting the same amino acid (p.Leu142Ser) has also been reported in ClinVar (RCV000650011). However, no further clinical information is reported. Given the molecular heterogeneity associated with Leigh syndrome, additional disease-causing candidate genes were also considered. The NDUFA1 variant c.94G $>C$ had been reported in two patients; one with a progressive neurodegenerative disease associated with complex I deficiency, and one with muscular hypotonia and lactic acidosis [4,11]. This association was supported by functional data in hamster cell lines confirming reduced complex I activity [4]. Nevertheless, segregation studies and analysis of recent MAF data among the general population suggests a non-pathogenic basis for the variant [12]. The TPK1 variants were therefore considered the most likely cause of disease in our family. The pathogenic effects were supported by segregation studies and a confirmed reduction in both plasma TPP concentration and hTPK1 steady state protein levels. Thus, despite significant advances in genomics, ancillary biochemical, and protein studies remain an important tool when investigating rare genetic diseases. 
A

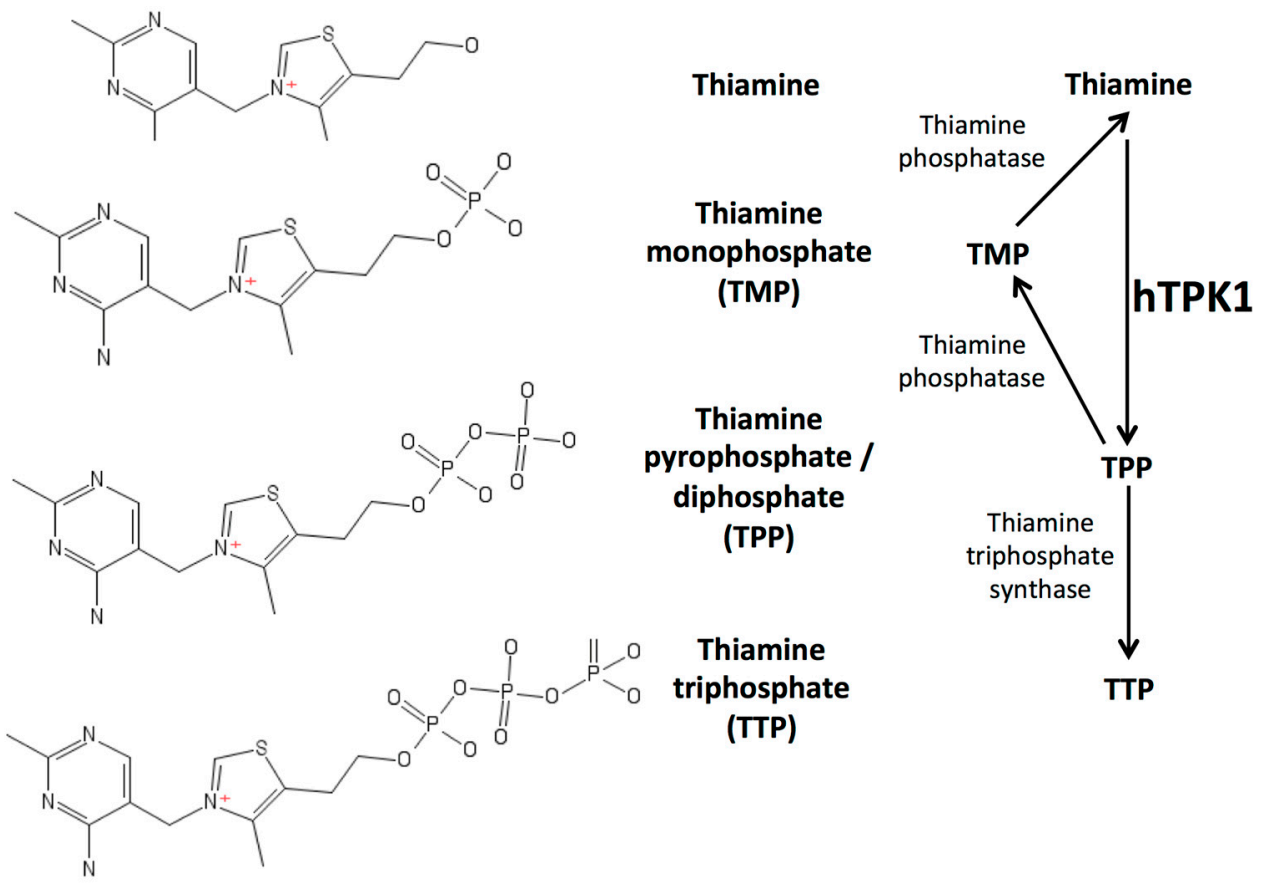

B

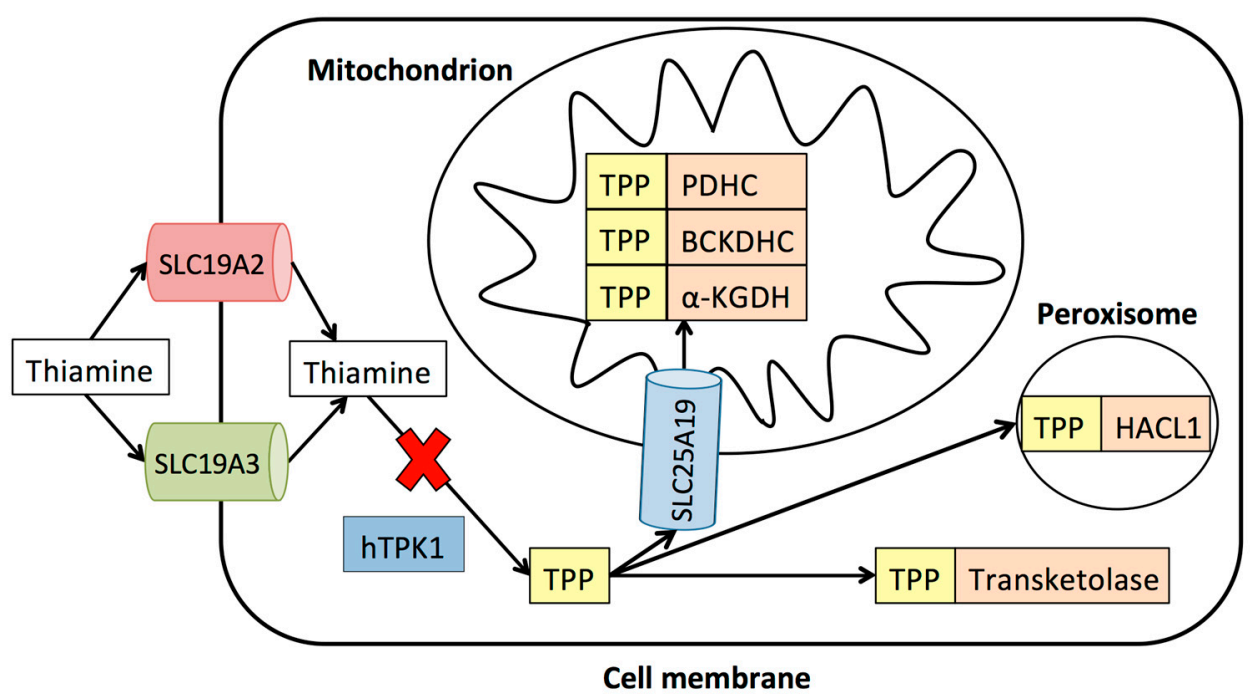

Figure 3. Thiamine structure and pathways. (A) Structure of different thiamine species and enzymatic pathways. Thiamine is phosphorylated to thiamine pyrophosphate (TPP) by thiamine pyrophosphokinase 1 (hTPK1). It can be further phosphorylated to thiamine triphosphate (TTP) by mitochondrial thiamine triphosphosphate synthase (ThTP synthase). It can also be dephosphorylated by thiamine phosphatase (Th phosphatase) to thiamine monophosphate (TMP) and thiamine. (B) Thiamine is absorbed in the small intestine and enters the cells using two transporters (encoded by SLC19A2/SLC19A3) where it is pyrophosphorylated to the active form (TPP) by hTPK1. TPP is transported into mitochondria by the SLC25A19 encoded carrier where it acts as a cofactor for three distinct dehydrogenases: (1) pyruvate dehydrogenase complex (PDHC); (2) branched-chain alfa keto acid dehydrogenase (BCKDH); and (3) alpha-ketoglutarate dehydrogenase ( $\alpha-\mathrm{KGDH})$. Outside of mitochondria, TPP acts a cofactor for Transketolase and 2-hydroxyacyl-Coa Lyase 1 (HACL1).

Despite the molecular heterogeneity exhibited by Leigh syndrome, TPP measurement represents a cost and time effective strategy when investigating suspected TPK1-related disease, with a high level 
of sensitivity; reported patients harbouring TPK1 mutations in whom whole blood or plasma TPP was measured (7/13) exhibited reduced concentrations (Table 1). There are a number of analytical methods for Vitamin B1 measurement described in the literature, with high performance liquid chromatography (HPLC) and fluorescence detection of oxidised thiamine derivatives being the most commonly used $[13,14]$. The availability of numerous laboratory techniques to measure TPP levels, in addition to variations in sample size, demographics, and dietary intake of control samples, has potentially contributed towards observed differences in reported TPP reference ranges, with the bottom end being between 63 and $105 \mathrm{nmol} / \mathrm{L}$ [15]. Regardless of the methodology, the most common derivative measured in blood is TPP given that this is the most abundant form of Vitamin B1 present. However, additional phosphorylated and unphosphorylated forms of thiamine are observed using HPLC chromatograms and can be identified with reference to standards, albeit at low abundance, and sometimes at the limit of quantitation [13,16]. Recently described methods [16-18], using HPLC with fluorescence or mass spectrometric detection, can quantify other forms of thiamine. These methods have potential utility during the investigation of inborn errors of thiamine metabolism using biological samples where other thiamine species are more abundant. For example, in cerebrospinal fluid, thiamine and TMP are more abundant than TPP. In our patient, a commercially available vitamin B1 HPLC kit (Chromsystems Instruments and Chemicals $\mathrm{GmbH}$, Munich, Germany) and an in-house derived reference range $(67-265 \mathrm{nmol} / \mathrm{L})$ was used to quantify TPP. The Chromsystems method extracts and derivatises vitamin B1 species from whole blood and then separates and detects these species by isocratic HPLC with fluorescence detection (https://www.chromsystems.com). Although this method is not designed to measure other forms of Vitamin B1, these can be observed as extra peaks in the chromatograms. This is particularly useful once the patient has commenced thiamine supplementation as prominent thiamine and TMP peaks are observed (Figure 4).

Table 1. Molecular and functional characteristics of reported TPK1 mutations.

\begin{tabular}{|c|c|c|c|c|c|}
\hline ID & TPK1 Mutations & Predicted Effect on Protein & TPP Levels & Western Blot & Reference \\
\hline P1 & c. $[148 \mathrm{~A}>\mathrm{C}] ;[501+4 \mathrm{~A}>\mathrm{T}]$ & p.[Asn50His];[Val119_Pro167del] & N/A & N/A & Mayr et al. [6] \\
\hline P2 & c. $[148 \mathrm{~A}>\mathrm{C}] ;[501+4 \mathrm{~A}>\mathrm{T}]$ & p.[Asn50His];[Val119_Pro167del] & $68 *(B)$ & $\downarrow$ hTPK1 & Mayr et al. [6] \\
\hline P3 & c. [119T $>C] ;[119 \mathrm{~T}>\mathrm{C}]$ & p.[Leu40Pro)];[Leu40Pro] & $50.4 *(\mathrm{~B})$ & $\downarrow$ hTPK1 & Mayr et al. [6] \\
\hline $\mathrm{P} 4$ & c. $[119 \mathrm{~T}>\mathrm{C}] ;[119 \mathrm{~T}>\mathrm{C}]$ & p.[Leu40Pro)];[(Leu40Pro] & N/A & N/A & Mayr et al. [6] \\
\hline P5 & c.[179_182delGAGA];[656A>G] & p.[Arg60LysfsTer52];[Asn219Ser] & $96.9 *(B)$ & $\downarrow$ hTPK1 & Mayr et al. [6] \\
\hline $\begin{array}{c}\text { P1 } \\
\text { (P76 in [5]) }\end{array}$ & c. $[604 \mathrm{~T}>\mathrm{G}] ;[604 \mathrm{~T}>\mathrm{G}]$ & p.[Trp202Gly];[Trp202Gly] & N/A & N/A & Fraser et al. [8] \\
\hline $\begin{array}{c}\text { P2 } \\
\text { (P77 in [5]) }\end{array}$ & c. $[604 \mathrm{~T}>\mathrm{G}] ;[604 \mathrm{~T}>\mathrm{G}]$ & p.[Trp202Gly];[Trp202Gly] & N/A & N/A & Fraser et al. [8] \\
\hline $\begin{array}{c}\text { P1 } \\
\text { (P79 in [5]) }\end{array}$ & c. $[479 \mathrm{C}>\mathrm{T}] ;[479 \mathrm{C}>\mathrm{T}]$ & p.[Ser160Leu];[Ser160Leu] & $60.9^{\wedge}(\mathrm{B})$ & Normal & Banka et al. [7] \\
\hline P2 & c. $[664 \mathrm{G}>\mathrm{C}] ;[664 \mathrm{G}>\mathrm{C}]$ & p.[Asp222His];[Asp222His] & $85.4^{\wedge}(\mathrm{B})$ & $\downarrow$ hTPK1 & Banka et al. [7] \\
\hline N/A & $\begin{array}{l}\text { c.[656A>G];deletion of exons } 3 \\
\text { and } 4 \text { on mRNA studies }\end{array}$ & p.[Asn219Ser];[?] & N/A & N/A & Invernizzi et al. [9] \\
\hline Proband & c. [119T>C];[119T>C] & p.[Leu40Pro];[Leu40Pro] & $32 \sim(\mathrm{P})$ & N/A & Mahajan et al. [10] \\
\hline Sister & c. $[119 \mathrm{~T}>\mathrm{C}] ;[119 \mathrm{~T}>\mathrm{C}]$ & p.[Leu40Pro];[Leu40Pro] & N/A & N/A & Mahajan et al. [10] \\
\hline P78 & c. $[365 \mathrm{~T}>\mathrm{C}] ;[365 \mathrm{~T}>\mathrm{C}]$ & p.[Ile122Thr];[Ile122Thr] & N/A & N/A & $\begin{array}{c}\text { Ortigoza-Escobar et al. } \\
{[5]}\end{array}$ \\
\hline Proband & c. $[258+1 \mathrm{G}>\mathrm{A}] ;[426 \mathrm{G}>\mathrm{C}]$ & p.[Leu142Phe];[?] & $35^{+}(\mathrm{B})$ & $\downarrow$ hTPK1 & Present study \\
\hline Brother & c. $[258+1 G>A] ;[426 G>C]$ & p.[Leu142Phe];[?] & N/A & N/A & Present study \\
\hline
\end{tabular}

Abbreviations: B, Blood; N/A, Not Available; P, Plasma; TPP, thiamine pyrophosphate. * Mayr et al. TPP reference range $132-271 \mathrm{nmol} / \mathrm{L} ;{ }^{\wedge}$ Banka et al. TPP reference range $132.2-271 \mathrm{nmol} / \mathrm{L} ; \sim$ Mahajan et al. TPP reference range 38-122 ng/L; † Present study TPP reference range 67-265 nmol/L. 


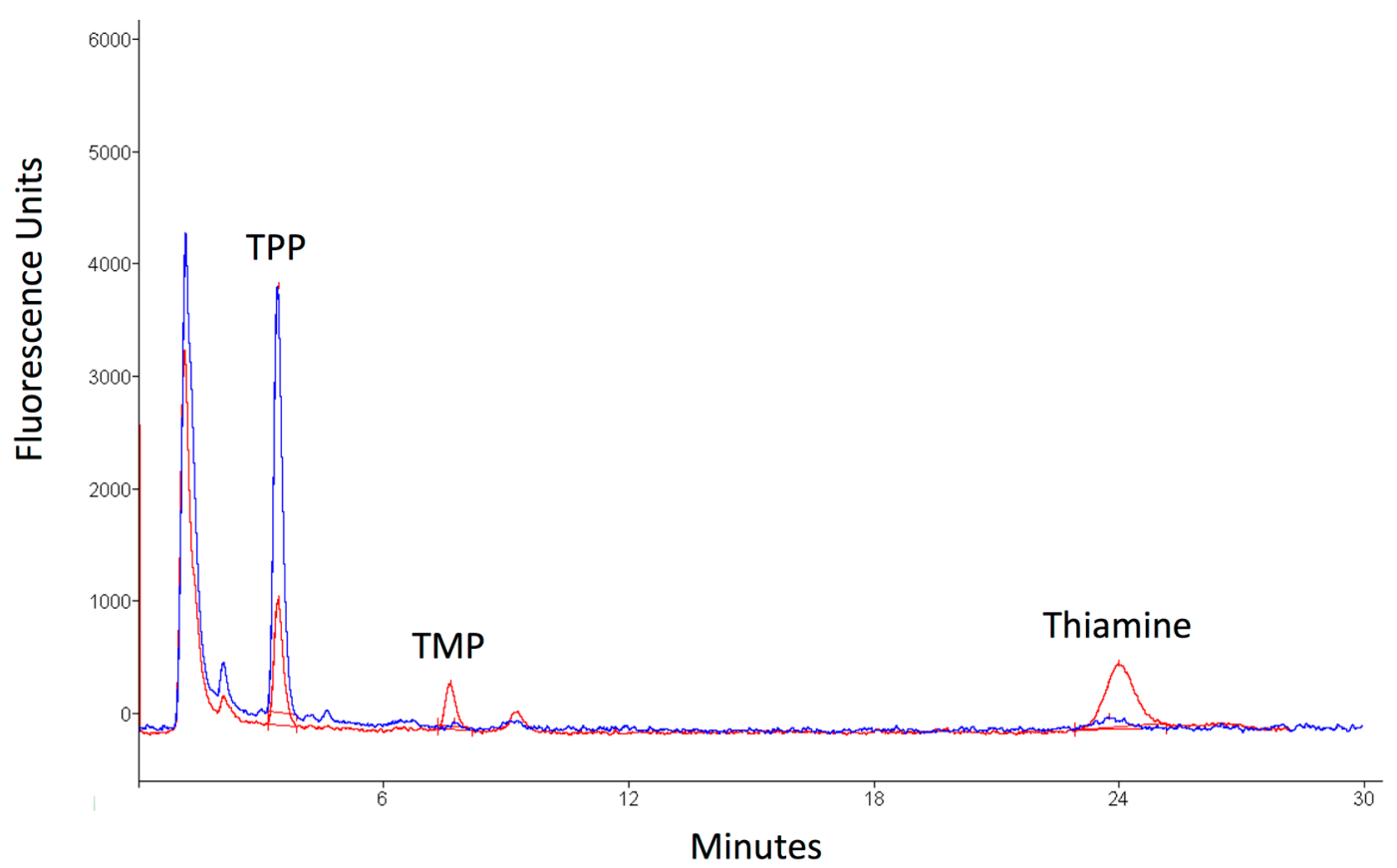

Figure 4. High performance liquid chromatography (HPLC) after 24 months of supplementation with thiamine. Red line: patient. Blue line: control. In patient there is a prominent peak for thiamine monophosphate (TMP) and unphosphorylated thiamine (thiamine) compared to control. These are caused by a reduced conversion of thiamine and TMP to thiamine diphosphate/pyrophosphate (TPP) for a deficient hTPK1 activity.

\section{Conclusions}

In conclusion, TPK1-related diseases pose a significant diagnostic challenge, given their clinical heterogeneity and overlap with other inherited metabolic and mitochondrial disorders. However, recognition is crucial to enable early therapeutic intervention with thiamine. TPP blood measurement represents a fast and cost-effective screening tool for suspected TPK1-related disorders to direct genetic testing and help filter potentially pathogenic variants generated by high-throughput sequencing methods. Research is ongoing to investigate our cohorts of children and adults with confirmed inborn errors of metabolism to evaluate the sensitivity and specificity of TPP measurement in this population.

Supplementary Materials: The materials are available online at http://www.mdpi.com/2077-0383/8/7/991/s1.

Author Contributions: Conceptualization, E.B., H.H., J.M., M.H., and R.P.; methodology, E.B., H.H., J.M., M.H.; investigation, E.B., S.P., R.F., O.P., A.P., C.W.; resources, S.H., R.Q., H.H., J.M., M.H.; data curation, A.P.; writing-original draft preparation, E.B., S.P., J.M., R.P.; writing-review and editing, E.B., S.P., S.H., R.Q., H.H., J.M., M.H., R.P.; visualization, E.B., S.P., R.F., R.P.; supervision, S.H., R.Q., H.H., J.M., M.H., R.P.

Funding: This research was funded by a Medical Research Council Centre grant (G0601943) and a European Community's Seventh Framework Programme (FP7/2007-2013), under grant agreement n² 2012-305121 “Integrated European -omics research project for diagnosis and therapy in rare neuromuscular and neurodegenerative diseases (NEUROMICS)". Part of this work was undertaken in the University College London Hospitals/University College London Queen Square Institute of Neurology sequencing facility, which received a proportion of funding from the Department of Health's National Institute for Health Research Biomedical Research Centres funding scheme. The clinical and diagnostic "Rare Mitochondrial Disorders" Service in London is funded by the UK NHS Highly Specialised Commissioners. R.D.S.P. is supported by a Medical Research Council Clinician Scientist Fellowship (MR/S002065/1). O.V.P. has received funding from the Lily Foundation. J.A.M was supported by the E-Rare project GENOMIT FWF I 2741-B26.

Acknowledgments: We would like to thank the family for their participation in the study.

Conflicts of Interest: The authors declare no conflict of interest. 


\section{Appendix A}

The study was performed under the ethical guidelines issued by our institution, with written informed consent obtained from participants for genetic studies.

\section{Whole Exome Sequencing}

Genomic DNA was extracted from peripheral leukocytes and prepared using Agilent SureSelect Target Enrichment Kit preparation guide and sequenced on HiSeq 2000/2500 sequencer (Illumina). The Illumina fastq sequencing data was mapped to the human reference assembly, hg19 (GRCh37; UCSC genome browser), and variants were called using GATK. Filtering of variants was performed using VarAft platform [19]. Variants with a MAF of $\geq 0.01$ in gnomAD, and 1000 Genomes databases and synonymous and deep intronic variants were excluded from further evaluation. Variants within genes encoding mitochondrial-localized proteins $(n=1158$, Mitocarta) were first considered. If no candidate mitochondrial disease-related genes were identified, variants in all coding genes were analyzed.

\section{Western Blot Analysis}

For western blot analysis, $10,000 \times g$ supernatants of fibroblast homogenates were used. Proteins were separated on a $10 \%$ acrylamide/bisacrylamide gel and transferred to nitrocellulose membranes (Biorad, Trans-Blot ${ }^{\circledR}$ Turbo $^{\mathrm{TM}}$ Mini Nitrocellulose Transfer Packs, \#1704158) using the Transblot Turbo System (Biorad). The membranes were washed in Tris-buffered saline (TBS) for 5 min, air-dried for $30 \mathrm{~min}$, then blocked for $30 \mathrm{~min}$ at room temperature in Western Blocking Reagent (Roche, \#11921673001), and dissolved in TBS-T (0.5\% Tween-20). After washing with TBS-T, the membranes were incubated with the primary antibody diluted in $1 x$ Western Blocking Reagent Solution in TBS-T. The western blot was incubated with the following antibodies: polyclonal rabbit anti-TPK1 (Sigma-Aldrich, HPA021849, overnight at $4{ }^{\circ} \mathrm{C}, 1: 1000$ ) and polyclonal rabbit anti-glucose-6-phosphate isomerase (Santa Cruz, sc-33777, $1 \mathrm{~h}$, room temperature, 1:1000). After washing with TBS-T, the membranes were incubated with the secondary antibody (Dako, Dako EnVision+ System-HRP Labelled Polymer Anti-Rabbit, K4003, $1 \mathrm{~h}$, room temperature, 1:100 in 1× Western Blocking Reagent Solution in TBS-T). For horse-radish peroxidase (HRP), detection the Lumi-Light ${ }^{\text {PLUS }}$ Western Blotting Substrate (Roche, \#12015196001) was used.

\section{References}

1. Brown, G. Defects of thiamine transport and metabolism. J. Inherit. Metab. Dis. 2014, 37, 577-585. [CrossRef] [PubMed]

2. Lek, M.; Karczewski, K.J.; Minikel, E.V.; Samocha, K.E.; Banks, E.; Fennell, T.; O’Donnell-Luria, A.H.; Ware, J.S.; Hill, A.J.; Cummings, B.B.; et al. Analysis of protein-coding genetic variation in 60,706 humans. Nature 2016, 536, 285-291. [CrossRef] [PubMed]

3. Calvo, S.E.; Clauser, K.R.; Mootha, V.K. MitoCarta2.0: An updated inventory of mammalian mitochondrial proteins. Nucleic Acids Res. 2016, 44, D1251-D1257. [CrossRef] [PubMed]

4. Potluri, P.; Davila, A.; Ruiz-Pesini, E.; Mishmar, D.; O’Hearn, S.; Hancock, S.; Simon, M.; Scheffler, I.E.; Wallace, D.C.; Procaccio, V. A novel NDUFA1 mutation leads to a progressive mitochondrial complex I-specific neurodegenerative disease. Mol. Genet. Metab. 2009, 96, 189-195. [CrossRef] [PubMed]

5. Ortigoza-Escobar, J.D.; Alfadhel, M.; Molero-Luis, M.; Darin, N.; Spiegel, R.; de Coo, I.F.; Gerards, M.; Taylor, R.W.; Artuch, R.; Nashabat, M.; et al. Thiamine deficiency in childhood with attention to genetic causes: Survival and outcome predictors. Ann. Neurol. 2017, 82, 317-330. [CrossRef] [PubMed]

6. Mayr, J.A.; Freisinger, P.; Schlachter, K.; Rolinski, B.; Zimmermann, F.A.; Scheffner, T.; Haack, T.B.; Koch, J.; Ahting, U.; Prokisch, H.; et al. Thiamine pyrophosphokinase deficiency in encephalopathic children with defects in the pyruvate oxidation pathway. Am. J. Hum. Genet. 2011, 89, 806-812. [CrossRef] [PubMed] 
7. Banka, S.; de Goede, C.; Yue, W.W.; Morris, A.A.; von Bremen, B.; Chandler, K.E.; Feichtinger, R.G.; Hart, C.; Khan, N.; Lunzer, V.; et al. Expanding the clinical and molecular spectrum of thiamine pyrophosphokinase deficiency: A treatable neurological disorder caused by TPK1 mutations. Mol. Genet. Metab. 2014, 113, 301-306. [CrossRef] [PubMed]

8. Fraser, J.L.; Vanderver, A.; Yang, S.; Chang, T.; Cramp, L.; Vezina, G.; Lichter-Konecki, U.; Cusmano-Ozog, K.P.; Smpokou, P.; Chapman, K.A.; et al. Thiamine pyrophosphokinase deficiency causes a Leigh Disease like phenotype in a sibling pair: Identification through whole exome sequencing and management strategies. Mol. Genet. Metab. Rep. 2014, 1, 66-70. [CrossRef]

9. Invernizzi, F.; Panteghini, C.; Chiapparini, L.; Moroni, I.; Nardocci, N.; Garavaglia, B.; Tonduti, D. Thiamine-responsive disease due to mutation of tpk1: Importance of avoiding misdiagnosis. Neurology 2017, 89, 870-871. [CrossRef]

10. Mahajan, A.; Sidiropoulos, C. TPK1 mutation induced childhood onset idiopathic generalized dystonia: Report of a rare mutation and effect of deep brain stimulation. J. Neurol. Sci. 2017, 376, 42-43. [CrossRef] [PubMed]

11. Mayr, J.A.; Bodamer, O.; Haack, T.B.; Zimmermann, F.A.; Madignier, F.; Prokisch, H.; Rauscher, C.; Koch, J.; Sperl, W. Heterozygous mutation in the X chromosomal NDUFA1 gene in a girl with complex I deficiency. Mol. Genet. Metab. 2011, 103, 358-361. [CrossRef] [PubMed]

12. $\mathrm{Xu}, \mathrm{J} . J . ;$ Ostergaard, E. Correspondence to: Heterozygous mutation in the $\mathrm{X}$ chromosomal NDUFA1 gene in a girl with complex I deficiency and A novel NDUFA1 mutation leads to progressive mitochondrial complex I-specific neurodegenerative disease. Mol. Genet. Metab. Rep. 2017, 13, 30. [CrossRef] [PubMed]

13. Edwards, K.A.; Tu-Maung, N.; Cheng, K.; Wang, B.; Baeumner, A.J.; Kraft, C.E. Thiamine Assays-Advances, challenges, and caveats. ChemistryOpen 2017, 6, 178-191. [CrossRef] [PubMed]

14. Lu, J.; Frank, E.L. Rapid HPLC measurement of thiamine and its phosphate esters in whole blood. Clin. Chem. 2008, 54, 901-906. [CrossRef] [PubMed]

15. Collie, J.T.B.; Greaves, R.F.; Jones, O.A.H.; Lam, Q.; Eastwood, G.M.; Bellomo, R. Vitamin B1 in critically ill patients: Needs and challenges. Clin. Chem. Lab. Med. 2017, 55, 1652-1668. [CrossRef] [PubMed]

16. Cheng, X.; Ma, D.; Fei, G.; Ma, Z.; Xiao, F.; Yu, Q.; Pan, X.; Zhou, F.; Zhao, L.; Zhong, C. A single-step method for simultaneous quantification of thiamine and its phosphate esters in whole blood sample by ultra-performance liquid chromatography-mass spectrometry. J. Chromatogr. B 2018, 1095, 103-111. [CrossRef] [PubMed]

17. Ortigoza-Escobar, J.D.; Molero-Luis, M.; Arias, A.; Oyarzabal, A.; Darin, N.; Serrano, M.; Garcia-Cazorla, A.; Tondo, M.; Hernandez, M.; Garcia-Villoria, J.; et al. Free-thiamine is a potential biomarker of thiamine transporter-2 deficiency: A treatable cause of Leigh syndrome. Brain 2016, 139, 31-38. [CrossRef] [PubMed]

18. Roelofsen-de Beer, R.; van Zelst, B.D.; Wardle, R.; Kooij, P.G.; de Rijke, Y.B. Simultaneous measurement of whole blood vitamin B1 and vitamin B6 using LC-ESI-MS/MS. J. Chromatogr. B 2017, 1063, 67-73. [CrossRef] [PubMed]

19. Desvignes, J.P.; Bartoli, M.; Delague, V.; Krahn, M.; Miltgen, M.; Beroud, C.; Salgado, D. VarAFT: A variant annotation and filtration system for human next generation sequencing data. Nucleic Acids Res. 2018, 46, W545-W553. [CrossRef] [PubMed]

(C) 2019 by the authors. Licensee MDPI, Basel, Switzerland. This article is an open access article distributed under the terms and conditions of the Creative Commons Attribution (CC BY) license (http://creativecommons.org/licenses/by/4.0/). 